\title{
THE DIMENSION OF THE SUPPORT OF A RANDOM DISTRIBUTION FUNCTION
}

BY J. R. KINNEY AND T. S. PITCHER

Communicated by Michel Loève, August 21, 1963

In their paper Random distribution functions (Bull. Amer. Math. Soc. 69 (1963), 548-551) L. E. Dubins and D. A. Freedman defined a random distribution function $F$ associated with a probability measure $\mu$ on the unit square $S$ whose values are distribution functions on $[0,1]$. To choose a value $F_{\omega}$ of $F$ they proceed as follows: Points $P(n, j)$ of $S$ are defined inductively for all $n$ and $j=0, \cdots, 2^{n}$ by setting $P(0,0)=(0,0), P(0,1)=(1,1), P(n+1,2 j)=P(n, j)$ and $P(n+1,2 j+1)$ equal to the image under the unique affine transformation carrying $S$ onto the rectangle $R(P(n, j), P(n, j+1))$ formed by the vertical and horizontal lines through $P(n, j)$ and $P(n, j+1)$ of a point $P^{*}(n+1,2 j+1)=\left(x^{*}(n, 2 j+1), y^{*}(n, 2 j+1)\right)$ chosen according to the distribution $\mu$ independently of the previous choices. They showed that $\bigcap_{n=1}^{\infty} \cup_{j=0}^{2^{n}} R(P(n, j), P(n, j+1))$ is the graph of a continuous monotone function $F_{\omega}(x)$ increasing from 0 to 1 on $[0,1]$, that is, a distribution function defining a measure $\widetilde{F}_{\omega}(E)$ $=\int_{E} d F_{\omega}(x)$ on measurable $E \subset[0,1]$. The inverse of $F_{\omega}(x)$ is also a continuous everywhere increasing function which we call $G_{\omega}(y)$ with corresponding measure $\widetilde{G}_{\omega}(E)$. Let

$$
\begin{aligned}
& I(n, j)=[x(n, j-1), x(n, j)], \\
& J(n, j)=[y(n, j-1), y(n, j)]
\end{aligned}
$$

and

$$
I(n, x)=I(n, j), J(n, x)=J(n, j) \text { for that } j \text { for which } x \in I(n, j) .
$$

$I(n, y)$ and $J(n, y)$ are defined similarly. Let $I^{*}(n, 2 j+\epsilon)$ $=\left[0, x^{*}(n, 2 j+1)\right]$ or $\left[x^{*}(n, 2 j+1), 1\right]$ and $J^{*}(n, 2 j+\epsilon)$ $=\left[0, y^{*}(n, 2 j+1)\right]$ or $\left[y^{*}(n, 2 j+1), 1\right]$ according as $\epsilon$ equals 0 or 1 . We shall write $|I|$ for the length of the interval $I$, and $h(a, b)$ for the function on $S$ given by $h(a, b)=a \log b+(1-a) \log _{2}(1-b)$. All logarithms are taken to the base 2. For any function $k(x, y)$ on $S$ we set

$$
E_{\mu}(k(x, y))=\int_{0}^{1} \int_{0}^{1} k(x, y) d \mu(x, y)
$$

and

$$
\sigma_{\mu}^{2}(k(x, y))=E_{\mu}\left(\left[k(x, y)-E_{\mu}(k(x, y))\right]^{2}\right) .
$$


TheOREM 1. (a) If $\sigma_{\mu}(h(y, x))<\infty$ then

$$
\lim _{n \rightarrow \infty} \frac{\log |I(n, x)|}{n}=E_{\mu}(h(y, x))
$$

almost everywhere $\left(\widetilde{F}_{\omega}\right)$ for almost all $\omega$.

(b) If $\sigma_{\mu}(h(y, y))<\infty$ then

$$
\lim _{n \rightarrow \infty} \frac{\log |J(n, x)|}{n}=E_{\mu}(h(y, y))
$$

almost everywhere $\left(\widetilde{F}_{\omega}\right)$ for almost all $\omega$.

(c) If $\sigma_{\mu}(h(x, x))<\infty$ then

$$
\lim _{n \rightarrow \infty} \frac{\log |I(n, y)|}{n}=E_{\mu}(h(x, x))
$$

almost everywhere $\left(\widetilde{G}_{\omega}\right)$ for almost all $\omega$.

(d) If $\sigma_{\mu}(h(x, y))<\infty$ then

$$
\lim _{n \rightarrow \infty} \frac{\log |J(n, y)|}{n}=E_{\mu}(h(x, y))
$$

almost everywhere $\left(\widetilde{G}_{\omega}\right)$ for almost every $\omega$.

In the proof we will need the following law of large numbers for martingales.

Lemma. If $f_{n}$ is $F_{n}$-measurable, where $F_{n}$ is an increasing sequence of $\sigma$-fields, $E\left(\left|f_{n}\right|\right)<\infty, E\left(\left|f_{n}\right|^{2}\right)=\sigma_{n}^{2}$ with $\sum_{n=1}^{\infty} \sigma_{n}^{2} / n^{2}<\infty$, and if $E\left(f_{n} \mid F_{n-1}\right)=0$ for all $n$ then $\lim _{n \rightarrow \infty} n^{-1} \sum_{j=1}^{n} f_{j}=0$ almost everywhere.

PROOF. $S_{n}=\sum_{j=1}^{n} f_{j} / j$ is a martingale, convergent to some limit $Z$ since $E\left(S_{n}^{2}\right) \leqq \sum_{j=1}^{\infty} \sigma_{j}^{2} / j^{2}$ for all $n$. Hence

$$
\begin{aligned}
\lim _{n \rightarrow \infty} \frac{1}{n} \sum_{j=1}^{n} f_{j} & =\lim _{n \rightarrow \infty} \frac{1}{n} \sum_{j=1}^{n} j\left(S_{j}-S_{j-1}\right)=\lim _{n \rightarrow \infty}\left(S_{n}-\frac{1}{n} \sum_{j=1}^{n-1} S_{j}\right) \\
& =Z-Z=0
\end{aligned}
$$

with probability one.

Proof OF TheOREM 1. The proofs of all sections of the theorem are the same so we confine ourselves to the first. Since

$$
\frac{1}{n} \log |I(n, x)|=\frac{1}{n} \sum_{k=1}^{n} \log \left|I^{*}(k, x)\right|
$$

the result will follow from the preceding lemma if we can show that $f_{k}=\log \left|I^{*}(k, x)\right|-E_{\mu}(h(y, x))$ satisfies $E_{Q}\left(f_{k} \mid F_{k-1}\right)=0$ and $E_{Q}\left(f_{k}^{2}\right)$ 
$=\sigma_{\mu}^{2}(h(y, x))$ where $F_{k}$ is the field generated by the $\left|I^{*}(l, x)\right|$ for $l \leqq k$ and $Q$ is the measure on $[0,1] x \Omega$ defined by $\int k(x, \omega) d Q$ $=E_{\omega}\left(\int_{0}^{1} k(x, \omega) d F_{\omega}(x)\right)$. Any $F_{k-1}$ measurable function has the form $g(x, \omega)=\sum_{j=1}^{2^{k-1}} g_{j} x_{j}(x, \omega)$ where $x_{j}(x, \omega)$ is 1 or 0 depending on whether $x$ is in $I(k-1, j)$ or not so

$E_{Q}\left(g f_{k}\right)=E_{\omega}\left(\sum_{j=1}^{2^{k-1}} g_{j} \int_{I(k-1, j)}\left(\log \left|I^{*}(k, u)\right|-E_{\mu}(h(y, x))\right) d F_{\omega}(u)\right)=0$

which shows that $E_{Q}\left(f_{k} \mid F_{k-1}\right)=0$. The verification that $E_{Q}\left(f_{\mathbf{k}}^{2}\right)$ $=\sigma_{\mu}^{2}(h(y, x))$ is straightforward.

Let $C_{\mu}=\left\{I_{j}\right\}$ be a set of intervals covering $E$ with $\max _{j}\left|I_{j}\right| \leqq \mu$. The $\alpha$-dimensional measure of $E$ is

$$
\Gamma_{\alpha}(E)=\lim _{\mu \rightarrow 0} \underset{C_{\mu}}{\text { g.l.b. }} \sum_{I_{i} \in C_{\mu}}\left|I_{j}\right|^{\alpha} .
$$

The Hausdorff-Besicovitch dimension of $E$ is

$$
\operatorname{dim} E=\inf \left(\beta \mid \Gamma_{\beta}(E)=0\right)=\sup \left(\beta \mid \Gamma_{\beta}(E)=\infty\right) .
$$

TheOREM 2. Under the hypotheses of Theorem 1, for almost all $\omega$, there exist sets $K_{\omega}, L_{\omega}$, with $\widetilde{F}_{\omega}\left(K_{\omega}\right)=\widetilde{G}_{\omega}\left(L_{\omega}\right)=1$, such that for any sets $A$ and $B$ with $\widetilde{F}_{\omega}(A)>0$ and $\widetilde{G}_{\omega}(B)>0$ we have

$$
\operatorname{dim}\left(K_{\omega} \cap A\right)=E_{\mu}\{h(y, y)\} / E_{\mu}\{h(y, x)\}
$$

and

$$
\operatorname{dim}\left(L_{\omega} \cap B\right)=E_{\mu}\{h(x, x)\} / E_{\mu}\{h(x, y)\} .
$$

PROOF. The proofs of the two statements are identical so we will prove only the first. Call the right-hand side of the first equation $\alpha$. We choose an $\omega$ in none of the exceptional sets of the first theorem. Then from the first two conclusions of the first theorem, there is a set $K_{\omega}$ with $\tilde{F}_{\omega}\left(K_{\omega}\right)=1$, such that $|J(n, x)|=|I(n, x)|{ }^{\alpha+o(1)}$ for all $x \in K_{\omega}$. For each $x$ in $K_{(\omega)}$ we choose that $I(n, x)$ with smallest $n$ such that $|I(n, x)|<\mu$ and $|J(n, x)|>|I(n, x)|^{\alpha+}{ }_{\epsilon}$. For $x_{1}, x_{2} \in I(n, x)$ the choice occurs at the same time so the $I(n, x)$ are disjoint and countable and cover $K_{\omega}$. Hence

$$
1=\int_{U_{I(n, x)}} d F_{\omega}(x)=\sum_{I(n, x)}|J(n, x)| \geqq \sum|I(n, x)|^{\alpha+\varsigma}
$$

so $\Gamma_{\alpha+\epsilon}\left(K_{\omega}\right) \leqq 1$, for every $\epsilon>0$, and hence $\operatorname{dim} K_{\omega} \leqq \alpha$. Let

$$
C\left(\epsilon_{1}, \epsilon_{2}\right)=\left[\left.x|| J(n, x)|>| I(n, x)\right|^{\alpha-\epsilon_{1}} \text { or }|I(n, x)|<2^{n\left[E \mu\{h(y, x)\}-\epsilon_{2}\right]}\right]
$$


for infinitely many $n$. Let $C_{\mu}\left(\epsilon_{1}, \epsilon_{2}\right)$ be the union of the intervals $I^{*}(n, x)$ covering $C\left(\epsilon_{1}, \epsilon_{2}\right)$ where for $x \in C\left(\epsilon_{1}, \epsilon_{2}\right) n$ is the smallest $n$ for which the conditions of $C\left(\epsilon_{1}, \epsilon_{2}\right)$ are satisfied with $\left|I^{*}(n, x)\right| \leqq \mu$. Since $\bigcap_{\mu \rightarrow 0} C_{\mu}\left(\epsilon_{1}, \epsilon_{2}\right)=C\left(\epsilon_{1}, \epsilon_{2}\right), \lim _{\mu \rightarrow 0} \widetilde{F}_{\omega}\left(C_{\mu}\left(\epsilon_{1}, \epsilon_{2}\right)\right)=0$. Suppose $\widetilde{F}_{\omega}(A)$ $=2 s$. Take $\mu$ so small that $\widetilde{F}_{\omega}\left(C_{\mu}\left(\epsilon_{1}, \epsilon_{2}\right)\right)<s$, let $A^{*}=A \cap c\left(C_{\mu}\left(\epsilon_{1}, \epsilon_{2}\right)\right)$ $\cap K_{\omega}$ where $c$ indicates complimentation, and set $M(x)$ $=\widetilde{F}\left(A^{*} \cap[0, x]\right) . M(x)$ is a monotone, continuous function, $M(1)>s$, and $M(x+h)-M(x-h)<(2 h)^{\alpha-\epsilon_{3}}$, where $\epsilon_{3}$ depends on the choice of $\epsilon_{1}$ and $\epsilon_{2}$. This happens since $M(x)$ increases only on $I(n, j)$ which fail to lie in $C_{\mu}\left(\epsilon_{1}, \epsilon_{2}\right)$. Hence, if $C_{\mu}=\left(I_{n}\right)$ is a covering of $A^{*}$ with $\left|I_{n}\right|<\mu$ then

$$
s \leqq \int_{A^{*}} d M(x)=\sum_{n} \int_{I_{n}} d M(x) \leqq \sum\left|I_{n}\right|^{\alpha-\epsilon_{3}} .
$$

Hence $\Gamma_{\alpha-\epsilon_{3}}\left(A^{*}\right)>s$. By adjusting $\epsilon_{1}$ and $\epsilon_{2}$, we can choose any $\epsilon_{3}>0$ so $\operatorname{dim} A^{*} \geqq \alpha$. Hence, with the previous inequality we have

$$
\alpha \leqq \operatorname{dim} A^{*} \leqq \operatorname{dim} A \cap K_{\omega} \leqq \operatorname{dim} K_{\omega} \leqq \alpha .
$$

LINCOLN LABORATORY ${ }^{1}$

${ }^{1}$ Operated with support from the U. S. Army, Navy and Air Force. 